\title{
EDITORIAL
}

\section{Practice, process and aesthetic reflection in electroacoustic music}

The motivation for this issue of Organised Sound sprang from a desire to mark out some territory for the relationship between practical processes relating to the composition of electroacoustic music, and the development of theoretical frameworks through and around those practices. A telling feature of the theoretical and aesthetic speculation in this field is that a substantial amount of it is carried out by active composers. The reasons for this are not likely to be straightforward and lie at least partly with the nature of the electroacoustic genre itself. In a medium that has to a considerable extent obviated the need for traditional or even nontraditional scores, analytical strategies must be based solely on aural perception of sound materials that are frequently very distant from anything that can be adequately represented on the page. As Barry Truax (1996) has eloquently noted, the complexity, the affinity with noise, and the referential openness of the sounding universe of electroacoustic sound make it controversial in terms of received notions of music and its traditional theoretical constructs. Yet despite the potentially rich vein of research problems that this invites, musicologists dedicated to, or even interested in the field remain rare. In a way, then, perhaps it is also because of this that composers - those familiar with music technology and with an instinct for its practice - have much to gain from reflection on the aesthetic multidimensionality of the medium. As David Keane (1989: 45), writing in 1979, said: 'When a potential composer of electronic music confronts his medium, it is immediately apparent that the initial obstacle is the infinite number of possibilities'.

So, is it now that creative practitioners' key position in the aesthetic literature of electroacoustic music is indicative of a situation where creative action alone may not be sufficient as intellectual sustenance amongst such a plethora of 'possibilities'? While Stravinsky once remarked, perhaps impishly, 'I do not see any means of explaining why I have chosen a certain note if whoever hears it does not already know why when he hears it' (Stravinsky and Craft 1960: 114), critical reflection on creative processes is nevertheless a crucial skill for creative individuals faced with decision-making in such a timbrally and structurally open field as that embraced by electroacoustic music. In fact, since the human/ machine relationship in electroacoustic music frequently creates situations where the application of a processing technique can throw up hugely unpredictable aural results, the composer must have analytical tools at his/ her disposal in order to find contexts for materials, to comprehend how to reshape them or to assign a musical value to them. Whilst the subjective experience of the composer is surely a catalyst for creative action, objectification of the outcomes, if not the nature of that experience, is a means of achieving the vision and overview that may be necessary to attain the autonomous aesthetic 'rightness' in a work that Stravinsky implied. And from a purely analytical/critical standpoint perhaps, even if words are no surrogate for a listener feeling an instinctive response of emotional or intellectual resonance with a specific piece, they may be a means of corroborating the spirit of a cluster of works. If we take the notion of 'theory' as emblematic of this aim, it is affirming to remember that locked in the etymology of the word is, after all, the action of taking the wider 'view' of the scene before us. Of course, the working methods of composers also vary, not just in terms of personal creative aspirations and cultural bases, but in terms of the nature of a relationship with the technology itself - since there coexist within the electroacoustic community composers who are content as 'end' users of extremely powerful sound processing technologies, and those who are compelled to build the tools they will use in the production of compositions ... to 'make something in order to make something', as it were. That distinction alone presents paths that may be quite different in technological emphasis, as programming and software design are in themselves creative activities, yet across these must be a core business of compositional technique that drives the construction, articulation and sensitivity to electroacoustic sound and its resulting formal designs. But there are aspects of compositional technique that are impossible to unpick with electroacoustic sound. In acousmatic music, for instance, the exact nature of signal processing techniques used and the nature of the assembly of sounds, such as the way stems and tracks contribute to a mix, often cannot be determined aurally. While this means that the analyst must very constructively grapple with the musical totality of the work, it may still leave intimate aspects of the composer's craft and decisionmaking impenetrable.

The present landscape is, then, one that sees a growing body of theoretical and speculative work, much of which is based around reflections on creative practice. Contributions to this issue acknowledge this in 
a variety of ways. Paul Rudy begins by questioning electroacoustic music's relationship to established perspectives and constructs of music. A key implication of his paper is that through mechanisms of common practice with timbre, a broader understanding of the 'musicality' of all sounds and the structures they create might be reached. Brian Kane's appraisal of Pierre Schaeffer's work as an expression of his concern with the practice of phenomenology makes us aware of the way in which musique concrète presented itself as a technologically enabled new practice. For not only were new sound materials made available for creative work, but the readiness of the human imagination to grasp this potential made viable a field that simultaneously demanded the physical fleshing out of its scope and potentials and the contextualisation of the practice through phenomenological observation. My own contribution presents a perspective on the notion of sound imagery, embracing the recognition of sound sources and the seeding of imaginative responses made possible through the maniplulation of sound with electroacoustic technology. A significant conceptual influence is acknowledged here in Denis Smalley's sketch of indicative fields and indicative networks. Placed alongside Brian Kane's discussion of the phenomenological origins of Schaeffer's researches, my paper offers a window on the widespread pragmatic reaction against artificially reductive approaches to the development of an aesthetic framework for electroacoustic music by deliberately exploiting the referential potentials of real-world sounds and the connotative implications of sound transformation. Denis Smalley, whose previous theoretical work on spectromorphology is strongly influenced by the work of Schaeffer, presents a discussion that significantly expands on his previous reflections on spatial morphology in electroacoustic music and charts a new course for spatially derived processes of analysis and awareness in practical creative contexts. Smalley argues that spatial awareness is a fundamental force in electroacoustic music, and this is used to link dimensions of temporal evolution, spectral structure and three-dimensional imaging of sound. The catalyst for many of the observations in this substantial article is a vividly portrayed experience of listening in a specific soundscape which is used to define aspects of spatial structure that are also considered in relation to a number of specific musical examples, including the author's own work. As such, Smalley reminds us that the listening process has become a crucial part of the practice of electroacoustic music, and not just in those who identify specifically as 'soundscape' composers.

If theoretical stances are to be effective, they spring from material evidence that validates them. But they also rely on their research communities to acknowledge them and their terms of reference - in short, to test theoretical ideas, not just through analysis of existing repertoire but also through the evaluation of descriptive models in creative action. In the light of a mounting body of theory pertaining to electroacoustic music, then, much could be gained by an assessment of the extent to which these writings have influenced composers. This dimension of the theory/practice relationship is at the heart of Daniel Schachter's discussion of his own practice in the composition of FlaX for real-time processed flute and pre-processed electroacoustic sounds. While drawing on Smalley's theoretical work, it is notable that he also bases much of his reflection on consideration of the role of Gestalt principles, allowing him to construct a unified and generalised view of his own creative processes as well as that of the performer engaging with electroacoustic sounds in a structurally variable musical environment. John Croft's contribution focuses on electroacoustically expanded notions of 'liveness'. Here he sketches a set of paradigms for the creation and reception of music for instruments and live electronics, based on a set of acute observations of the behavioural and interactive relationships that can be projected. Sophy Smith investigates interaction at the level of co-operative exchange between multiple participants in the creative process, namely UK-based hiphop turntable teams. While the insight offered by this paper into a collaborative and real-time creative process is valuable in itself, the framework constructed for evaluative comparison of the teams studied also illustrates one general model for the mapping and analysis of creative processes with technology. Concluding this issue, and not specifically addressing the issue's theme, Roger Dean and Freya Bailes's reflections on Locke's An Essay Concerning Human Understanding, points to the ways in which the seeds of a coherent vision of an aesthetic of organised sound might exist within the complexity of human experience, however remote from the specifics of our contemporary technologies.

The spectrum of papers in this issue covers a broad range of perspective from which to view the practice/ theory landscape. This includes composers demonstrating aspects of their creative and procedural thinking, reflections of the intensified approach to listening that music technology has enabled (demanded, even), and perspectives on the electroacoustic music's situation in relation to the roots of traditional musical practice. The charged immediacy of sound production and manipulation in the early twenty-first century electroacoustic music studio does obviously present composers with ever more responsive and speedier production environments. At a superficial level this might be good news for those wishing increase their rate of musical output, but it also enhances our scope to investigate ever more thoroughly, through practice, the subtleties, variations and inflexions available in the shaping of sound with digital audio tools. In turn, the triangulation of theory derived from the essence of practice, and 
practice informed by acknowledgement and testing of theoretical constructs, must surely offer the possibility of a clear path through the infinite number of possibilities'.

John Young Music, Technology and Innovation Research Centre De Montfort University Leicester, UK E-mail:jyoung@dmu.ac.uk

\section{REFERENCES}

Keane, D. 1989. Some practical aesthetic problems of electronic music composition. In R. J. Heifetz (ed.) On the Wires of Our Nerves: The Art of Electroacoustic Music. Cranbury, NJ / London: Associated University Presses.

Stravinsky, I., and Craft, R. 1960. Memories and Commentaries. London: Faber.

Truax, B. 1996. Soundscape, acoustic communication and environmental sound composition. Contemporary Music Review 15(1): 49-65. 